Aim of the study: Cervical cancer is the second most common malignancy in women worldwide. Everolimus displays direct effects on growth and proliferation of cancer cells via inhibition of mammalian target of rapamycin (mTOR) protein, which is known to be associated with drug resistance. In this study, we aimed to investigate the effects of everolimus, gemcitabine, and paclitaxel in terms of cell viability and mRNA expression levels of GRP78, CCND1, CASP2, and BCL2 genes.

Material and methods: HeLa cells were treated with different doses of everolimus, gemcitabine, and paclitaxel. Cell viability was assessed using MTT assay, and obtained dose response curves were used for the calculations of inhibitory concentration (IC) values. At the end of the treatment times with selected doses, RNA isolation and CDNA synthesis were performed Finally, GRP78, CCND1, CASP2, and $B C L 2$ genes mRNA expression levels were analysed using quantitative PCR. Results: The IC50 value of everolimus was $0.9 \mu \mathrm{M}$ for 24-hour treatment. Moreover, the IC50 value of gemcitabine and paclitaxel was found to be around $18.1 \mu \mathrm{M}$ and $7.08 \mu \mathrm{M}$, respectively. Everolimus, gemcitabine, and paclitaxel treatments alone did not change the GRP78, CCND1, BCL2 and CASP2 mRNA expression levels significantly. However, combined treatment of everolimus and paclitaxel significantly reduced BCL2 and CCND1 mRNA expression $(p<0.05)$. In contrast, this combination did not change GRP78 and CASP2 mRNA expression levels $(p>0.05)$.

Conclusions: Down-regulation of CCND1 and BCL2 expression may be an important mechanism by which everolimus increases the therapeutic window of paclitaxel in cervical cancers.

Key words: everolimus, gene expression, HeLa cells, paclitaxel, unfolded protein response, GRP78, BCL2, CCND1, CASP2.

Contemp Oncol (Pozn) 2016; 20 (1): 28-32 DOI: $10.5114 /$ wo.2016.58498

\section{Reduced BCL2 and CCND1 mRNA expression in human cervical cancer HeLa cells treated with a combination of everolimus and paclitaxel}

\author{
Akin Yilmaz ${ }^{1}$, Ebru Alp ${ }^{2}$, H. Ilke Onen³, Sevda Menevse ${ }^{3}$
}

${ }^{1}$ Department of Medical Biology, Faculty of Medicine, Hitit, University, Corum, Turkey 2Department of Medical Biology, Faculty of Medicine, Giresun University, Giresun, Turkey ${ }^{3}$ Department of Medical Biology and Genetics, Faculty of Medicine, Gazi University, Besevler, Ankara, Turkey

\section{Introduction}

Cervical cancer is the second most common malignancy in women worldwide, and it remains a leading cause of cancer-related death for women in developing countries [1]. Although, the most pivotal aetiological agent is human papillomavirus (HPV) in the development of cervical cancer, extra molecular changes are also defined in the pathogenesis of cervical cancer [1, 2]. In recent years, autophagy has appeared as a substantial biological mechanism in targeting human cancers, including carcinoma of the cervix [3].

Autophagy, also known as type II programmed cell death, is one of the homeostatic and evolutionarily conserved processes found in cells. Activation of autophagy leads to the targeting of cellular proteins and organelles to lysosomes for degradation [4]. By impeding the increase of impaired proteins and organelles, autophagy prevents tumour formation. On the other hand, it can also encourage the growth of developed tumours via acting as a cell survival mechanism. Unfolded protein response (UPR) after endoplasmic reticulum (ER) stress and the mammalian target of rapamycin (mTOR) signalling pathway are two potent regulators of autophagy [5].

Endoplasmic reticulum stress, which results from accumulation of unfolded, misfolded, or damaged proteins in ER lumen, activates autophagy directly through upregulation of GRP78 and through mechanisms downstream of GRP78 release of the other UPR signal transducers [6]. GRP78 was shown to be obligatory for autophagosome formation by gene knockdown experiment [7].

mTOR is activated downstream of phosphoinositide 3-kinase (PI3K)/ v-akt murine thymoma viral oncogene homolog (AKT) pathway. By associating several proteins, mTOR forms two different protein complexes: mTOR complex 1 (mTORC1) and mTOR complex 2 (mTORC2). Predominantly, mTORC1 regulates cell growth, cell cycle progression, macromolecule biosynthesis, and autophagy. However, mTORC2 controls cell survival and metabolism, as well as organisation of cytoskeleton [7, 8].

Unusual activation of PI3K/AKT/mTOR signalling pathway has frequently been found in several cancers from different origins, including cervical tumours [10]. It has been associated with resistance to chemotherapeutics and worst prognosis [11]. Upregulation of PI3K gene in cervical cancer cell line (HeLa) was shown, and administration of PI3K inhibitor (LY294002) blocked cell proliferation [12].

It is known that a natural mTOR inhibitor named rapamycin and its analogues (rapalogues), including everolimus, selectively target mTORC1 to stimulate autophagy [5]. Studies have revealed striking clinical therapeutic 
results for rapalogues in several tumours as single-agent therapies or in combination with other therapeutics. Thus, a combination of mTOR inhibitors and classical chemotherapeutics was suggested as an effective approach to treat cancer, based on remarkable findings obtained from both preclinical studies and clinical observations [13, 14].

mTOR inhibition displays direct effects on the growth and proliferation of cancer cells via downregulation of genes having roles in cell cycle progression, such as cyclin D1 (CCND1) and cyclin dependent kinase 4 (CDK4) [15]. There is also consistent evidence that the AKT/mTOR signalling pathways may be associated with resistance to taxanes and other drugs acting on microtubules [16]. For this reason, in vivo combinations of everolimus with paclitaxel showed additive and synergistic effects in tumour models [17].

Some cytoskeletal disrupting agents, including paclitaxel, have potent anticancer activities against a variety of tumours from different origins [18]. These agents kill cancer cells eventually by inducing apoptotic pathway, but the mechanism is not clearly understood. However, some studies have underlined the proapoptotic function of caspase-2 (CASP2) $[19,20]$ and the antiapoptotic function of B-cell CLL/lymphoma 2 (BCL2) molecules in cell death induced by cytoskeletal disruption [21-23].

In this study, we aimed to investigate the effects of everolimus, gemcitabine, and paclitaxel on cervical cancer cell line (HeLa) viability. Moreover, changes of relative mRNA expression levels of GRP78, CCND1, CASP2, and $B C L 2$ genes were also investigated after administration of these agents, alone or in combination, to the cells by real-time PCR analysis.

\section{Material and methods}

\section{Cell culture}

HeLa cells (HÜKÜK \#90061901) were obtained from the Animal Cell Culture Collection in the Foot and Mouth Disease Institute, Turkey. Cells were cultured in RPMI1640 medium supplemented with 10\% foetal bovine serum (FBS), $200 \mathrm{mM}$ L-glutamine, $100 \mathrm{lU} / \mathrm{ml}$ penicillin, and $100 \mu \mathrm{g} / \mathrm{ml}$ streptomycin (all obtained from HyClone, USA) in a humidified atmosphere containing $5 \% \mathrm{CO}_{2}$ at $37^{\circ} \mathrm{C}$.

\section{Cell viability assay}

Cell viability was assessed using MTT (3-[4,5-dimethylthiazol-2-yl]-2,5 diphenyl tetrazolium bromide) assay. Cells were seeded into 96 -well plates at a density of $1 \times$ $10^{4}$ cells/well in medium containing $1 \%$ FBS and incubat- ed overnight to attach. Then the cells were treated with $10 \%$ FBS medium containing various concentrations of everolimus (25-500 nM), gemcitabine $(0.125-8 \mu \mathrm{M})$, and paclitaxel (25-500 nM) and incubated for 24 hours (all obtained from Sigma-Aldrich, USA). At the end of the treatment times MTT mixture was added to each well and incubated for an additional four hours. Then the medium was discarded and formazan crystals were dissolved in dimethyl sulfoxide (DMSO). The absorbance values were measured at $570 \mathrm{~nm}$ using a Spectramax M3 microplate reader (Molecular Devices, USA). Control wells for absorbance readings contained DMSO and MTT mixture. All experiments were repeated five times for each dose and the mean absorbance values were calculated. The inhibitory concentration values $\left(I C_{10}, I C_{25}, I C_{50}, I C_{75}\right.$, and $\left.I C_{90}\right)$ for each agent were calculated from the dose-response curves by means of GraphPad Software PRISM 5 (GraphPad Software, USA).

After analysis of the response of the cells to the agents, selected doses of agents ( $400 \mathrm{nM}$ for everolimus, $8 \mu \mathrm{M}$ for gemcitabine, and $100 \mathrm{nM}$ for paclitaxel) were given to the cells as single agent or in dual combinations (i.e. everolimus + gemcitabine and everolimus + paclitaxel) and incubated for 24 hours to perform mRNA expression analysis.

\section{RNA isolation and cDNA synthesis}

At the end of the incubation time, total RNA was isolated using High Pure RNA Isolation Kit (Roche Diagnostics, Mannheim, Germany) according to the manufacturer's protocol. The quantity and quality of the isolated RNA was measured using a NanoDrop ND-1000 spectrophotometer (NanoDrop Technologies, USA). One microgram of total RNA from each sample was reverse transcribed with random hexamers using a Transcriptor First Strand CDNA Synthesis Kit (Roche Diagnostics, Mannheim, Germany) according to the manufacturer's instructions. The cDNA reaction was performed in an Eppendorf Mastercycler EP gradient S thermal cycler (Eppendorf, Hamburg, Germany).

\section{Real-time quantitative PCR}

Real-time PCR was carried out using a Light Cycler ${ }^{\circledR}$ 480 (Roche Diagnostics GmbH, Mannheim, Germany). Light Cycler ${ }^{\circledR} 480$ Probe Master reaction mix in combination with human Universal Probe Library (UPL) probes was used to determine gene expression levels (Roche Diagnostics, Mannheim, Germany). Expression levels of the target genes were normalised to mRNA level of glyceraldehyde-3-phosphate dehydrogenase (GAPDH) gene. Intron-spanning primers and specific probes for GRP78,

Table 1. Primer sequences and probe numbers used in real-time quantitative PCR for the detection of target mRNAs.

\begin{tabular}{|c|c|c|c|c|}
\hline Gene & $\begin{array}{c}\text { mRNA accession } \\
\text { number }\end{array}$ & Forward primer & Reverse primer & $\begin{array}{l}\text { UPL probe } \\
\text { number }\end{array}$ \\
\hline GRP78 & NM_005347 & 5'-ACTGTTACAATCAAGGTCTATGAAGG-3' & 5'- CAAAGGTGACTTCAATCTGTGG-3' & 15 \\
\hline$B C L 2$ & NM_000633 & 5'-AGTACCTGAACCGGCACCT-3' & 5'-GGCCGTACAGTTCCACAAA-3' & 75 \\
\hline CASP2 & NM_032982 & 5'-CGCCATCTATGGTGTGGAT-3' & 5'-CAGTTGGCGTTGTCAAAGAG-3' & 78 \\
\hline GAPDH & NM_002046 & 5'-AGCCACATCGCTCAGACAC-3' & 5'-GCCCAATACGACCAAATCC-3' & 60 \\
\hline
\end{tabular}




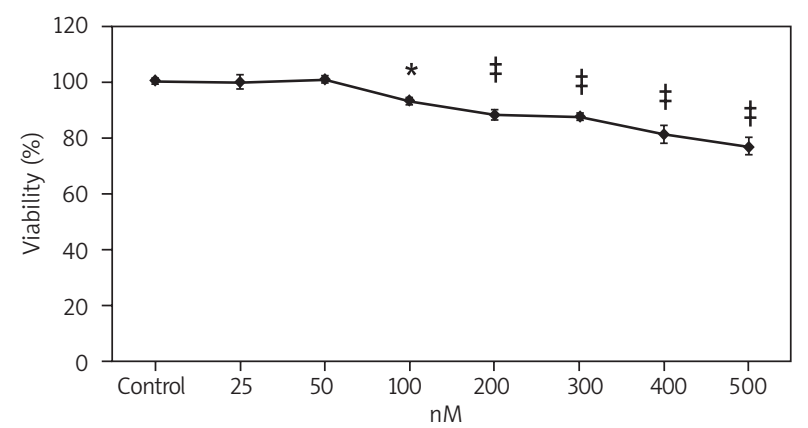

* indicates $p<0.05, \neq$ denotes $p<0.001$

Fig. 1. Effects on cell viability of everolimus treatment on HL-60 cells measured by MTT assay. The results are expressed as a percentage of control. Data shown are the means of five independent experiments

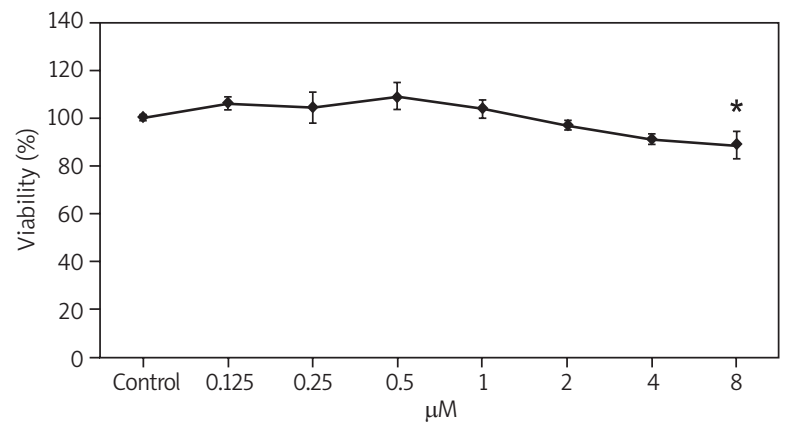

* indicates $p<0.05$

Fig. 2. Effects of gemcitabine treatment on HeLa cell viability measured by MTT assay. The results are expressed as a percentage of control. Data shown are the means of five independent experiments

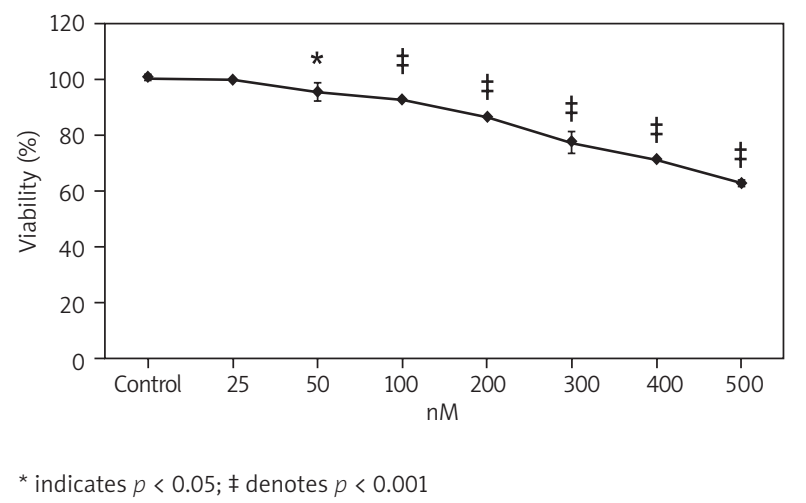

Fig. 3. Effects of paclitaxel treatment on HeLa cell viability measured by MTT assay. The results are expressed as a percent of control. Data shown are the means of five independent experiments
CCND1, CASP2, BCL2, and GAPDH transcripts were selected using the online Universal Probe Library Assay Design Centre (http://www.universalprobelibrary.com). Primers and probes used in the real-time quantitative PCR for the detection of target mRNAs were given in Table 1. The quantitative polymerase chain reaction ( $\mathrm{PPCR}$ ) conditions were as follows: after initial denaturation at $94^{\circ} \mathrm{C}$ for 5 minutes, 45 cycles consisting of $95^{\circ} \mathrm{C}$ for 15 seconds, and $60^{\circ} \mathrm{C}$ for 20 seconds, and finally samples were cooled down to $40^{\circ} \mathrm{C}$. The qPCR was repeated three times for each gene.

\section{Statistical analysis}

Changes of GRP78, CCND1, CASP2, and BCL2 mRNA expression levels were compared by Relative Expression Software Tool (REST 2009 v2.013) [24]. Other parameters were analysed by one-way analysis of variance (ANOVA) with Tukey's post hoc tests using SigmaStat 3.5 software. Data were expressed as the mean \pm SD (standard deviation) from a representative experiment. $P$ values lower than 0.05 were considered as significant.

\section{Results}

The dose-response curve for everolimus-treated HeLa cells is given in Fig. 1. Everolimus did not show a significant growth inhibitory effect on HeLa cells at 25- and 50-nM concentrations for 24 hours $(p>0.05)$. However, cell viability was significantly affected at $100 \mathrm{nM}$ everolimus concentration $(p<0.05)$. Furthermore, at doses higher than $100 \mathrm{nM}$, everolimus remarkably decreased cell viability ( $p<0.001$ ) and $76 \%$ of the cells were determined as viable at the highest dose used in our study (i.e. $500 \mathrm{nM}$ ). As shown in Table 2 , the $I C_{50}$ value of everolimus was calculated as $907.5 \mathrm{nM}$ (or $0.9 \mu \mathrm{M}$ ) for 24-hour treatment.

No significant viability changes were found for gemcitabine-treated cells at doses between 0.125 and $4 \mu \mathrm{M}$. On the other hand, the highest gemcitabine dose tested in our study significantly affected HeLa cell viability $(p<0.05)$, and $88 \%$ of the cells were determined as viable after treatment with $8 \mu \mathrm{M}$ gemcitabine (Fig. 2). The $I C_{50}$ value of gemcitabine was found to be around $18.1 \mu \mathrm{M}$ for this treatment duration (Table 2).

The most striking anti-proliferative effect was seen in paclitaxel-treated cells when compared to the everolimusand gemcitabine-treated cells. Significant dose-dependent decreases in viability were obtained at $50 \mathrm{nM}(p<0.05)$ and $\geq 100 \mathrm{nM}(p<0.001)$ paclitaxel concentrations. After incubation with $500 \mathrm{nM}$ paclitaxel for 24 hours, HeLa cell viability decreased to $62 \%$ compared to untreated cells (Fig. 3). The $I C_{50}$ value of paclitaxel was found to be about $708 \mathrm{nM}$ (or $7.08 \mu \mathrm{M}$ ) as calculated from the concentration-response curve (Table 2).

Table 2. Calculated IC values of everolimus, gemcitabine, and paclitaxel

\begin{tabular}{|c|c|c|c|c|c|}
\hline & $\mathrm{IC}_{10}$ & $\mathrm{IC}_{25}$ & $\mathrm{IC}_{50}$ & $\mathrm{IC}_{75}$ & $\mathrm{IC}_{90}$ \\
\hline Everolimus (nM) & 224.9 & 451.7 & 907.5 & 1823.1 & 3662.5 \\
\hline Gemcitabine $(\mu M)$ & 4.9 & 9.4 & 18.1 & 35 & 67.7 \\
\hline Paclitaxel (nM) & 141.3 & 316.4 & 708.8 & 1587.7 & 3556.3 \\
\hline
\end{tabular}


In order to analyse whether treatment with these agents alone or in combination for 24 hours affected key molecules having roles in unfolded protein response, cell cycle regulation, and antiapoptotic and proapoptotic genes including GRP78, CCND1, BCL2, and CASP2, we evaluated changes in the expression of these genes using quantitative real-time PCR. The results of mRNA expression analysis of the GRP78, CCND1, BCL2, and CASP2 genes are given in Fig. 4. We found that everolimus, gemcitabine, and paclitaxel alone did not significantly change the GRP78, CCND1, BCL2, and CASP2 mRNA expression levels. Moreover, combined treatment with everolimus and gemcitabine did not significantly change mRNA expressions ( $p>0.05)$. However, combined treatment of everolimus and paclitaxel significantly reduced BCL2 and CCND1 mRNA expression $(p<0.05)$. Conversely, this combination did not change GRP78 and CASP2 mRNA expression levels ( $p>0.05$ ). CCND1 and BCL2 mRNA expression were decreased to 3.2-fold and 2.2-fold, respectively, at $400 \mathrm{nM}$ everolimus plus $100 \mathrm{nM}$ paclitaxel combination as compared to the control cells $(p<0.05)$.

\section{Discussion}

Due to its antiapoptotic property, stress induction of GRP78 mRNA represents an important pro-survival component of the UPR. Several agents have been shown to induce GRP78 mRNA expression in cancer cells. Activation of mRNA synthesis of the GRP78 gene in cell culture systems is considered as a sign for the presence of ER stress and the beginning of the UPR [25]. However, we did not show any significant changes for this gene in HeLa cells treated with everolimus alone or in combination with gemcitabine or paclitaxel. From this point of view, our results suggest that all the agents used in our study may not activate unfolded protein response at analysed concentrations.

The association of PI3K/mTOR signalling pathway in the development of cervical cancer was shown by analysing cervical tumour and non-neoplastic tissues. Researchers also indicated effective antigrowth effect of a PI3K inhibitor, LY294002, on HeLa cells [12]. In another study, PI3K inhibition was found to produce significant radiosensitisation in cervical cancer cells [26]. Similarly, rapamycin was shown to enhance the chemosensitivity of CaSki cells, derived from a cervical epidermoid carcinoma metastatic to the small bowel mesentery, to paclitaxel and cisplatin [27, 28]. In gastric cancer cell lines, everolimus had synergistic effects with 5-fuorouracil. Moreover, the growth inhibitory effect of everolimus was found to be related to G1 phase arrest and decreased expression of cyclins A, D, and E [29]. In HeLa cells, mTOR inhibition with rapamycin caused cell cycle arrest and increased accumulation of cells in GO/ G1 [30]. In ovarian cancer cell lines (SK-OV-3 and IGROV1), treatment with rapamycin or everolimus was associated with a decrease in the expression of CCND1 and CDK4, as well as G1 arrest. Moreover, BCL2 expression was found to be a marker of resistance to programmed cell death induced by mTOR inhibitor in ovarian cancer cells [31]. In our study, we found that administration of everolimus, gemcitabine, and paclitaxel alone to the HeLa cells did not

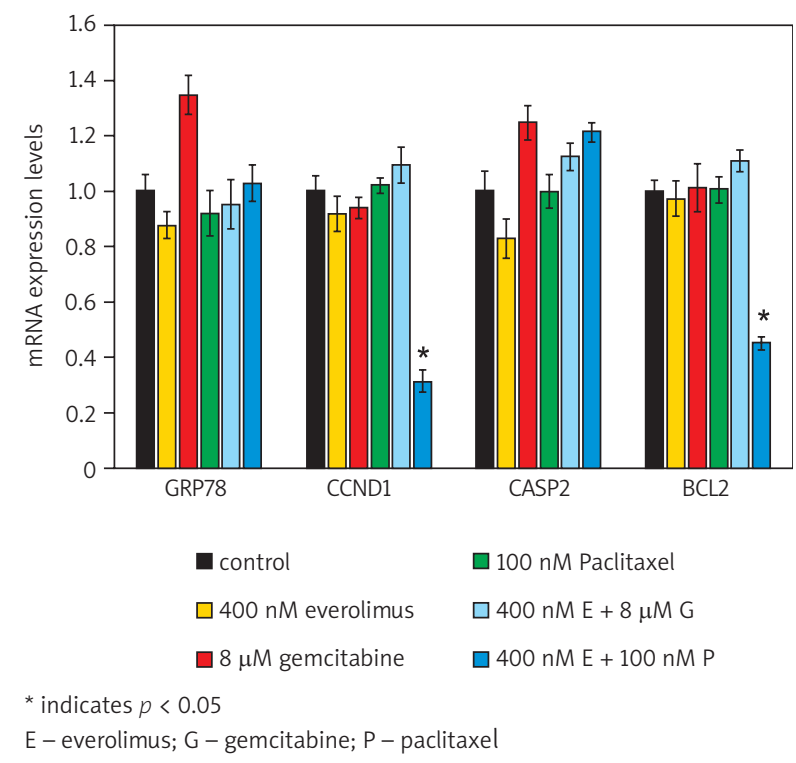

Fig. 4. Quantitative RT-PCR analysis results of GRP78, BCL2, CASP2, and CCND1 mRNAs of HeLa cells treated with drugs for 24 hours. Gene expression levels were normalised to GAPDH mRNA expression level

significantly change the mRNA expression of the CCND1 and BCL2 genes. However, combined treatment with everolimus and paclitaxel significantly diminished CCND1 mRNA expression (about three-fold) (Fig. 4). With respect to CCND1 in cell cycle regulation and its role in G1/S transition, this reduction can result in G1/S arrest.

Caspase- 2 is considered as one of the initiator caspases, but its biological roles remain a matter of controversy [32]. CASP2 gene deregulation in some cell lines suppress cell death pathway in response to antineoplastic drugs. The extent of caspase-2 involvement in apoptotic cell death depends on several of factors, including the nature of the cytotoxic agents, the origin of the cell type, and the intracellular abundance of caspase-2 relative to that of other pro-apoptotic molecules [33]. There have been a limited number of studies investigating the roles of CASP2 in cancer development, including cervical cancer.

Significant elevation in caspase- 2 activity was found in human cervical intraepithelial lesions, cervical cancer samples, and in the serum of donors, as compared to controls [32]. In our study, we showed the presence of CASP2 mRNA expression in untreated HeLa cells. Moreover, mRNA expression pattern of CASP2 did not significantly change after treatment with everolimus, gemcitabine, and paclitaxel alone or in combination. Although, CASP2 was reported to be responsible for cell death induced by cytoskeletal poisons [20], we could not show any change in its mRNA levels after administration of paclitaxel alone or in combination with gemcitabine or everolimus.

In summary, mRNA expression of anti-apoptotic and proliferative genes did not change after paclitaxel alone, but BCL2 and CCND1 mRNAs expression decreased after a combination of paclitaxel with everolimus. Down-regulation of CCND1 and BCL2 expression may be an important mechanism by which everolimus increases the therapeutic window of paclitaxel in cervical cancer cells. A better un- 
derstanding of the molecular response of the cells to both newly developed agents and chemotherapeutics will help to improve the therapy options of patients with cancer.

\section{The authors declare no conflict of interest.}

\section{References}

1. Paavonen J. Human papillomavirus infection and the development of cervical cancer and related genital neoplasias. Int J Infect Dis 2007; 11 (Suppl2): S3-9.

2. Yang HJ. Aberrant DNA methylation in cervical carcinogenesis. Chin J Cancer 2013; 32: 42-8.

3. Pandey S, Chandravati C. Autophagy in cervical cancer: An emerg ing therapeutic target. Asian Pac J Cancer Prev 2012; 13: 4867-71.

4. Degenhardt K, Mathew R, Beaudoin B, et al. Autophagy promotes tumor cell survival and restricts necrosis, inflammation, and tum origenesis. Cancer Cell 2006; 10: 51-64.

5. Yang ZJ, Chee CE, Huang S, Sinicrope FA. The role of autophagy in cancer: therapeutic implications. Mol Cancer Ther 2011; 10: 1533 41.

6. Benbrook DM, Long A. Integration of autophagy, proteasomal degradation, unfolded protein response and apoptosis. Exp Oncol 2012; 34: 286-97.

7. Li J, Ni M, Lee B, Barron E, Hinton D, Lee A. The unfolded protein response regulator GRP78/BiP is required for endoplasmic reticu lum integrity and stress-induced autophagy in mammalian cells. Cell Death Differ 2008; 15: 1460-71.

8. Chiang GG, Abraham RT. Targeting the mTOR signaling network in cancer. Trends Mol Med 2007; 13: 433-42.

9. Laplante M, Sabatini DM. mTOR signaling in growth control and disease. Cell 2012; 149: 274-93.

10. Feng W, Duan X, Liu J, Xiao J, Brown RE. Morphoproteomic evidence of constitutively activated and overexpressed mTOR pathway in cervical squamous carcinoma and high grade squamous intraepithelial lesions. Int J Clin Exp Pathol 2009; 2: 249-60.

11. Guertin DA, Sabatini DM. Defining the Role of mTOR in Cancer. Cancer Cell 2007; 12: 9-22.

12. Zhang XY, Zhang HY, Zhang PN, Lu X, Sun H. Elevated phosphatidylinositol 3-kinase activation and its clinicopathological significance in cervical cancer. Eur J Obstet Gynecol Reprod Biol 2008; 139: 237-44.

13. Lopiccolo J, Blumenthal G, Bernstein W, Dennis P. Targeting the $\mathrm{PI} 3 \mathrm{~K} / \mathrm{Akt} / \mathrm{mTOR}$ pathway: Effective combinations and clinical considerations. Drug Resist Updat 2008; 11: 32-50.

14. Meric-Bernstam F, Gonzalez-Angulo AM. Targeting the mTOR signaling network for cancer therapy. J Clin Oncol 2009; 27: 2278-87.

15. Vignot S, Faivre S, Aguirre D, Raymond E. mTOR-targeted therapy of cancer with rapamycin derivatives. Ann Oncol 2005; 16: 525-37.

16. Campone M, Levy V, Bourbouloux E, et al. Safety and pharmacokinetics of paclitaxel and the oral MTOR inhibitor everolimus in advanced solid tumours. Br J Cancer 2009; 100: 315-21.

17. O'Reilly T, McSheehy PMJ, Wartmann M, Lassota P, Brandt R, Lane $H A$. Evaluation of the mTOR inhibitor, everolimus, in combination with cytotoxic antitumor agents using human tumor models in vitro and in vivo. Anticancer Drugs 2011; 22: 58-78.

18. Bhalla KN. Microtubule-targeted anticancer agents and apoptosis. Oncogene 2003; 22: 9075-86.

19. Ho LH, Read SH, Dorstyn L, Lambrusco L, Kumar S. Caspase-2 is required for cell death induced by cytoskeletal disruption. Oncogene 2008; 27: 3393-404.

20. Kumar S. Caspase 2 in apoptosis, the DNA damage response and tumour suppression: enigma no more? Nat Rev Cancer 2009; 9: 897-903.

21. Benimetskaya L, Miller P, Benimetsky, et al. Inhibition of potentially anti-apoptotic proteins by antisense protein kinase C-alpha (Isis 3521) and antisense bcl-2 (G3139) phosphorothioate oligodeoxynucleotides: relationship to the decreased viability of T24 bladder and PC3 prostate cancer cells. Mol Pharmacol 2001; 60: 1296-307.
22. Jansen B, Schlagbauer-Wadl H, Brown BD, et al. Bcl-2 antisense therapy chemosensitizes human melanoma in SCID mice. Nat Med 1998; 4: 232-34.

23. Zangemeister-Wittke U, Leech SH, Olie RA, et al. A novel bispecific antisense oligonucleotide inhibiting both bcl-2 and bcl-xL expression efficiently induces apoptosis in tumor cells. Clin Cancer Res 2000; 6: 2547-55.

24. Pfaffl MW, Horgan GW, Dempfle L. Relative expression software tool (RESTC) for group-wise comparison and statistical analysis of relative expression results in real-time PCR. Nucleic Acids Res 2002; 30: e36.

25. Li J, Lee AS, Stress induction of GRP78/BiP and its role in cancer. Curr Mol Med 2006; 6: 45-54.

26. Lee CM, Fuhrman CB, Planelles V, et al. Phosphatidylinositol 3-kinase inhibition by LY294002 radiosensitizes human cervical cancer cell lines. Clin Cancer Res 2006; 12: 250-56.

27. Faried LS, Faried A, Kanuma T, Nakazato T, Tamura T, Kuwano H, Minegishi T. Inhibition of the mammalian target of rapamycin (mTOR) by rapamycin increases chemosensitivity of CaSki cells to paclitaxel. Eur J Cancer 2006; 42: 934-47.

28. Faried LS, Faried A, Kanuma T, Sano T, Nakazato T, Tamura T, Kuwano $\mathrm{H}$, Minegishi T. Predictive and prognostic role of activated mammalian target of rapamycin in cervical cancer treated with cisplatin-based neoadjuvant chemotherapy. Oncol Rep 2006; 16: 57-63.

29. Lee $\mathrm{KH}, \mathrm{Hur} \mathrm{HS}$, Im SA, et al. RAD001 shows activity against gastric cancer cells and overcomes 5-FU resistance by downregulating thymidylate synthase. Cancer Lett 2010; 299: 22-8.

30. Ji J, Zheng PS. Activation of mTOR signaling pathway contributes to survival of cervical cancer cells. Gynecol Oncol 2010; 117: 103-8.

31. Aguirre D, Boya P, Bellet D, et al. Bcl-2 and CCND1/CDK4 expression levels predict the cellular effects of mTOR inhibitors in human ovarian carcinoma. Apoptosis 2004; 9: 797-805.

32. Babas E, Ekonomopoulou MT, Karapidaki I, Doxakis A, Betsas G, lakovidou-Kritsi Z. Indication of participation of caspase-2 and caspase-5 in mechanisms of human cervical malignancy. Int J Gynecol Cancer 2010; 20: 1381-85.

33. Zhivotovsky B, Orrenius S. Caspase-2 function in response to DNA damage. Biochem Biophys Res Commun 2005; 331: 859-67.

\section{Address for correspondence}

Dr. Akin Yilmaz, Assoc. Prof. Department of Medical Biology

Faculty of Faculty of Medicine Hitit University

Corum, 1930, Turkey

e-mail: akinyilmaz@hitit.edu.tr; akinyilmaz2002@yahoo.com

Submitted: 27.03 .2014

Accepted: 23.01 .2015 